\section{Augmentation of Antioxidant Constituents by Drought Stress to Roots in Leafy Vegetables}

\author{
Ryohei Koyama $^{1,2}$, Hiromichi Itoh ${ }^{3}$, Syuji Kimura ${ }^{2}$, Ai Morioka ${ }^{2}$, \\ and Yuichi Uno ${ }^{1,4}$
}

Additional InDEX words. dehydration, L-ascorbic acid, nitrate, plant factory, polyphenol, rhizosphere, soilless culture

Summary. Plants can synthesize some antioxidants, including L-ascorbic acid (AsA) and polyphenol, in response to environmental stresses. Antioxidants detoxify reactive oxygen species in plants and also aid in human health. In this study, we demonstrate that a novel hydroponic treatment can increase leafy vegetable nutritional quality without retarding growth. Leaf lettuce (Lactuca sativa) was grown hydroponically and subjected to rhizosphere drought stress by lowering the water level in the solution tub before harvesting. Appropriate drought stress using this method could increase AsA, polyphenol, and sugar content by $24 \%, 50 \%$, and $17 \%$, respectively, and decrease nitrate nitrogen content by $18 \%$ without reducing yield. Similar effects of drought stress on AsA content were observed in four other plant species. This hydroponic method has a universal potential to increase leafy vegetable quality without reducing yield in controlled environments such as plant factories.

$\mathrm{I}$ n recent years, consumer health concerns have increased market demand for safe and nutritious foods. Leafy vegetables are a rich source of calcium, iron, $\beta$-carotene, AsA (vitamin C), and dietary fiber. They contain more micronutrients per mouthful than any other food (Gupta and Prakash, 2008). Plants can synthesize some antioxidants including, for example, AsA, glutathione, $\alpha$-tocopherol (vitamin E), and polyphenol to protect themselves against oxidative damage caused by environmental stressors (Blokhina et al., 2003; Romani et al., 2002). These compounds evolved to detoxify reactive oxygen species in plants, but they also show beneficial activity against some human diseases related to oxidative damage and aging (Fusco et al., 2007; Iwai, 2008; Nicolle et al., 2004). Multiple studies have reported increasing antioxidant levels in plants via intentional environmental stress, such as drought, temperature, and high light intensity (Hamilton and Fonseca,

We thank Dr. Noboru Inagaki, Dr. Michio Kanechi, and Miss Noriko Ohyanagi for their valuable suggestions and fruitful discussions.

${ }^{1}$ Department of Bioresource Science, Graduate School of Agricultural Science, Kobe University, 1-1 Rokko, Kobe 657-8501, Japan

${ }^{2}$ Nihon Yamamura Glass Co., Ltd., 15-1 Nishimukojimacho, Amagasaki, Hyogo 660-8580, Japan

${ }^{3}$ Department of Agricultural Engineering, Graduate School of Agricultural Science, Kobe University, 1-1 Rokko, Kobe 657-8501, Japan

${ }^{4}$ Corresponding author. E-mail: yuno@kobe-u.ac.jp.
2010; Johkan et al., 2010; Oh et al., 2009; Sharma and Dubey, 2005). However, these techniques to improve plant quality are hard to implement in agricultural fields because of the risk of overstress, which has adverse effects on plant growth and yield (Hidaka et al., 2008). A plant factory is an efficient and stable system in which plants are cultivated in a controlled environment year-round. The products are chemicalfree, fresh, and clean (Takatsuji, 2010). For the success of industries based on hydroponically grown plants, it is not only important to increase yields but also to improve quality. Improvements to quality attributes such as flavor and/or nutritional value can differentiate hydroponically grown products from field-grown products. The environmental control techniques in a plant factory make it possible to alter these characteristics by treating plants with moderate and consistent levels of stress.
Hydroponic culture is currently the main cultivation method in plant factories. In hydroponic culture, some types of stress can be easily introduced to the roots. Stress to the roots causes declines in root absorption and leaf photosynthesis and results in both osmotic and oxidative stress to the whole plant (Kitano et al., 2008). For example, irrigating tomato (Solanum lycopersicum) plants with salt water is commonly practiced and produces high-quality fruits enriched in sugar and minerals (Cuartero and Fernández-Muñoz, 1999). Spinach (Spinacia oleracea) plants that were stressed with cold treatment of their roots had increased levels of beneficial sugar, AsA, and ferric ions $\left(\mathrm{Fe}^{2+}\right)$ in their leaves as well as decreased levels of nitrate ions $\left(\mathrm{NO}_{3}{ }^{-}\right)$ and oxalic acid, which are harmful substances (Hidaka et al., 2008). However, in these reports the growth of the aerial parts of these stressed plants declined, highlighting the challenges that overstressing plants pose to commercial cultivation. An alternative cultivation method is necessary to increase quality without reducing yield.

Ideally, an environmental stress treatment for commercial production would have a low cost. Drought stress by suddenly exposing part of the roots to air can be practiced simply by lowering water levels in hydroponic culture and does not require extra equipment, such as ultraviolet lamps, or alteration of other conditions in the culture room, equipment, or solutions. In addition, this method might allow easier fine-tuning of stress levels than alternative methods. New cultivation systems that use less water than conventional hydroponics systems have been developed as an economical, low-maintenance method for growing lettuce (Kratky, 1993, 2004). In this system, roots are exposed to airspace

\begin{tabular}{llll}
\hline $\begin{array}{l}\text { Units } \\
\begin{array}{l}\text { To convert U.S. to SI, } \\
\text { multiply by }\end{array}\end{array}$ & U.S. unit & SI unit & $\begin{array}{l}\text { To convert SI to } \\
\text { U.S., multiply by }\end{array}$ \\
\hline 29,574 & $\mathrm{fl} \mathrm{oz}$ & $\mu \mathrm{L}$ & $3.3814 \times 10^{-5}$ \\
29.5735 & $\mathrm{fl} \mathrm{oz}$ & $\mathrm{mL}$ & 0.0338 \\
2.54 & inch $(\mathrm{es})$ & $\mathrm{cm}$ & 0.3937 \\
1 & $\mathrm{mmho} / \mathrm{cm}$ & $\mathrm{mS} \cdot \mathrm{cm}^{-1}$ & 1 \\
28.3495 & $\mathrm{oz}$ & $\mathrm{g}$ & 0.0353 \\
28,350 & $\mathrm{oz}$ & $\mathrm{mg}$ & $3.5274 \times 10^{-5}$ \\
0.1 & $\mathrm{ppm}$ & $\mathrm{mg} / 100 \mathrm{~g}$ & 10 \\
2.2417 & $\mathrm{ton} / \mathrm{acre}$ & $\mathrm{Mg} \cdot \mathrm{ha}^{-1}$ & 0.4461 \\
$\left({ }^{\circ} \mathrm{F}-32\right) \div 1.8$ & ${ }^{\circ} \mathrm{F}$ & ${ }^{\circ} \mathrm{C}$ & $\left(1.8 \times{ }^{\circ} \mathrm{C}\right)+32$
\end{tabular}


during the entire growth period and absorb nutrients from a noncirculating nutrient solution in a tub. However, to date, there have been no studies on optimization of conditions or short-term treatments to increase the nutritional quality of plants grown in this system. In this study, we demonstrate that a limited drought stress treatment to part of the root mass enhances the nutritional attributes of leafy vegetables without damaging growth.

\section{Materials and methods}

Plant material. We used five plant species in this study: 'Frillice' leaf lettuce (Snow Brand Seed Co., Hokkaido, Japan), 'Kyoumizore' potherb mustard [Brassica rapa (Takii \& Co., Kyoto, Japan)], 'Orai' spinach (Takii \& Co.), 'Nanako' turnip leaf [B. rapa (Takii \& Co.)], and 'Kikujiro' crown daisy [Glebionis coronaria (Takii \& Co.)].

Hydroponics. Seeds were sown in growing medium cubes made of urethane and irrigated with OtsukaSA nutrient solution (Otsuka AgriTechno Co., Osaka, Japan) containing (in micromole per liter) 6731 nitrogen, 569 phosphorus, 3928 potassium, 1580 calcium, 577 magnesium, 4 manganese, 6 boron, 19 iron, 0.2 copper, 0.5 zinc, and 0.1 molybdenum. The nutrient solution had a $\mathrm{pH}$ of 6.0 and electrical conductivity (EC) of $1.0 \mathrm{mS} \cdot \mathrm{cm}^{-1}$. Five days after sowing, seedlings were transferred to a hydroponic system using OtsukaSA nutrient solution with EC of 1.8 $\mathrm{mS} \cdot \mathrm{cm}^{-1}$. EC and $\mathrm{pH}$ were kept stable during the culture period by exchanging the nutrient solution every day. The maximum depth of the solution tubs was $6.0 \mathrm{~cm}$, and the effective volumetric capacity was $\approx 10.5 \mathrm{~L}$. Vegetables were cultivated for an additional $23 \mathrm{~d}$ (leaf lettuce), $13 \mathrm{~d}$ (potherb mustard), $17 \mathrm{~d}$ (spinach), $16 \mathrm{~d}$ (turnip leaf), or $31 \mathrm{~d}$ (crown daisy) and subjected to the drought stress immediately before harvest. Plants were grown under a $14 \mathrm{~h}$ light/10 h dark photoperiod with light provided by cool-white fluorescent lamps $\left(\approx 60 / 140 \mu \mathrm{mol} \cdot \mathrm{m}^{-2} \cdot \mathrm{s}^{-1}\right.$ photosynthetic photon flux during germination/cultivation). The nutrient solution was continuously aerated with an air pump. The thermostatic room and the solutions were maintained at $20^{\circ} \mathrm{C}$.
DROUGHT STRESS TREATMENT. Adult plants of leaf lettuce were subjected to drought stress to the rhizosphere by lowering the water level in the solution tub (Fig. 1). To determine the ideal drought conditions, the water level was reduced (i.e., increased the airspace in the rhizosphere) to 0 (control), 0.5, 1.0, 2.0, and $4.0 \mathrm{~cm}$ for $7 \mathrm{~d}$. There were six replicate plants in a solution tub for each treatment. When seedlings were 35-d old (including the $7 \mathrm{~d}$ of drought treatment), the aboveground tissues of seedlings were harvested and used to analyze plant growth and nutrient contents. Optimal stress conditions were determined by measuring leaf fresh weight and levels of AsA as described below. In a separate experiment, we varied the duration of the drought treatment by lowering the water to a fixed level of $4.0 \mathrm{~cm}$ of airspace for 0 (control), 3, 7, 9, 12 , and $14 \mathrm{~d}$ and then we evaluated leaf quality as before. We also measured the concentrations of polyphenolic compounds, $\mathrm{NO}_{3}{ }^{-}$nitrogen, and sugar in the lettuce leaves. The optimum drought conditions, as determined by AsA levels in lettuce, were then applied to the four other vegetable species.

ANALYSIS OF PLANT GROWTH AND NUTRIENT CONTENT. We measured both the fresh and dry weights of the aerial plant parts. Dry weight was measured after drying the plants in an oven at $80^{\circ} \mathrm{C}$ for a minimum of 48 h. Leaf water content (LWC) was calculated with the following formula: $\mathrm{LWC}=($ leaf fresh weight leaf dry weight $) /($ leaf fresh weight $) \times$ 100.

Total AsA content was determined with analysis strips (Ascorbic Acid Test; Merck, Darmstadt, Germany) using a reflectometer (RQflex plus, Merck) according to Tabata et al. (2001). All fresh leaf samples from lettuce were homogenized in an equal volume of $5 \%(\mathrm{w} / \mathrm{v})$ metaphosphoric acid in a blender, then centrifuged at $7740 g_{\mathrm{n}}$ for $1 \mathrm{~min}$. Crude samples of the supernatant were measured for AsA. Leaf samples from potherb mustard, spinach, turnip leaf, and crown daisy were harvested, immediately frozen in liquid nitrogen, and stored at $-80{ }^{\circ} \mathrm{C}$ until analysis.

We used the Folin-Denis method to determine total polyphenolic

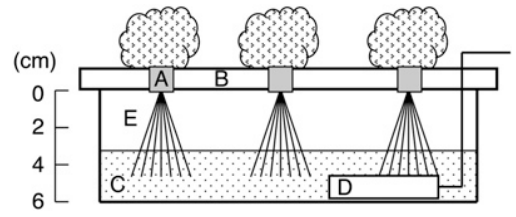

Fig. 1. Schematic view of the hydroponic system used to induce drought stress to roots of leafy vegetables. Five-day-old seedlings in growing medium cubes (A) were transferred to a hydroponic system. The seedlings were supported by a plastic foam bed (B) and irrigated with a nutrient solution $(C)$ that was aerated with an air stone (D). After $23 \mathrm{~d}$, seedlings were exposed to stress treatment by lowering the water level from 0 to $4 \mathrm{~cm}(\mathrm{E})$ in the solution tub for various periods before harvesting. Conditions for growth and treatments were as follows: $20^{\circ} \mathrm{C}\left(68.0^{\circ} \mathrm{F}\right), 14-\mathrm{h}$ light/10-h dark photoperiod with light provided by cool-white fluorescent lamps (photosynthetic photon flux of $60 / 140 \mu \mathrm{mol} \cdot \mathrm{m}^{-2} \cdot \mathrm{s}^{-1}$ for germination/cultivation); $1 \mathrm{~cm}=$ 0.3937 inch.

compounds in frozen samples, according to Matsumoto et al. (2009). Samples $(500 \mathrm{mg})$ were macerated in $5 \mathrm{~mL}$ of $80 \%(\mathrm{v} / \mathrm{v})$ ethanol, incubated at $4{ }^{\circ} \mathrm{C}$ for $2 \mathrm{~h}$ and then centrifuged at $800 g_{\mathrm{n}}$ for $5 \mathrm{~min}$. Then, we added both $500 \mu \mathrm{L}$ of $1 / 2$ Folin-Ciocalteu reagent (MP Biomedicals, Solon, $\mathrm{OH}$ ) and $10 \%(\mathrm{w} / \mathrm{v})$ sodium carbonate solution to $500 \mu \mathrm{L}$ of supernatant, vortexed the solution, and let it stand for $60 \mathrm{~min}$ at room temperature. Finally, the absorbance was determined at $700 \mathrm{~nm}$ using an absorption spectrophotometer (ultraviolet-160A; Shimadzu Corp., Kyoto, Japan). A quercetin standard curve was prepared from a freshly prepared quercetin (Wako Pure Chemical Industries, Osaka, Japan) solution in $80 \%(\mathrm{v} / \mathrm{v})$ ethanol.

We measured the concentration of $\mathrm{NO}_{3}{ }^{-}$nitrogen with analysis strips (Nitrate Test, Merck) using a reflectometer (RQflex plus) according to Sunaga et al. (1999). Crushed samples of $500 \mathrm{mg}$ were diluted with distilled water and centrifuged at $7740 g_{\mathrm{n}}$ for $1 \mathrm{~min}$. Crude samples of the supernatant were measured for $\mathrm{NO}_{3}^{-}$.

To measure sugar concentration, fresh leaf samples were homogenized with $80-\mathrm{mL}$ water in a blender and centrifuged at $7740 g_{\mathrm{n}}$ for $3 \mathrm{~min}$. The 
sugar concentration in the supernatant was measured with a refractometer (PAL-1; Atago Co., Tokyo, Japan).

Data for all variables showed a normal distribution and were compared by one-way analysis of variance followed by two sets of comparisons with the unpaired Student's $t$ test or multiple comparisons with the Tukey-Kramer method.

\section{Results}

To determine the optimum level of stress for accumulating AsA, adult lettuce plants were subjected to drought stress to the rhizosphere $28 \mathrm{~d}$ after sowing, as shown in Fig. 1. We varied the level of stress from mild to severe by lowering the water level in the solution tub (i.e., increasing the airspace) by differing amounts. The average root length of lettuce plants was $30.9 \mathrm{~cm}$ at the beginning of the stress treatment, so an average of between $2 \%$ (with $0.5-\mathrm{cm}$ airspace) and $13 \%$ (with $4-\mathrm{cm}$ airspace) of the total root length was exposed to air. Although the taproot was not altered in different treatments, exposed parts of lateral roots were withered in stressed plants. Figure 2 shows the changes in total AsA content in response to 7-d drought treatments (4-cm airspace in the solution tub). Total AsA content in the leaves increased with increasing airspace in the rhizosphere. A significant increase in AsA was observed at $2 \mathrm{~cm}$ of airspace and above, with maximum AsA levels found at $4 \mathrm{~cm}$ airspace. Leaf fresh weight ranged from 112 to $119 \mathrm{~g}$, but there were no significant differences among the treatments (data not shown). As the yield rate was $100 \%$ in lettuce, yield was calculated to be $\approx 39 \mathrm{Mg} \cdot \mathrm{ha}^{-1}$. Although we could not test the effects of lowering the water levels by more than $4 \mathrm{~cm}$ because our tubs were only $6 \mathrm{~cm}$ deep, we anticipate that leaf fresh weight would decline at lower water levels based on the nonsignificant trend in our data.

Fixing the airspace to $4 \mathrm{~cm}$ in depth, the optimum drought period was investigated (Fig. 3). Total AsA content increased steadily for the first $7 \mathrm{~d}$, reaching its highest levels at Days 7 and 9 (Fig. 3, closed circles). The AsA on those days was 1.24 times higher than that of the control (Day $0)$, which showed a significant difference. After $9 \mathrm{~d}$ of drought treatment, total AsA content began to decline, reaching the same levels as the control

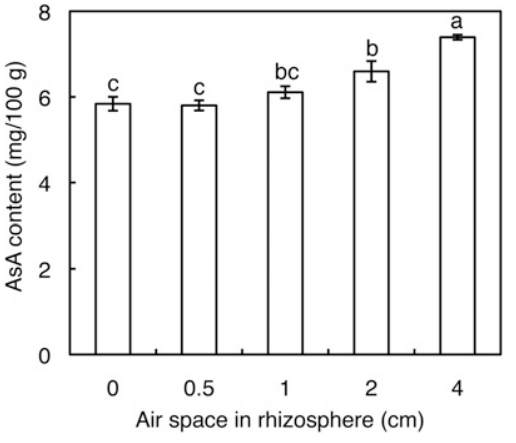

Fig. 2. Effects of intensity of drought stress to roots on total L-ascorbic acid (AsA) content in lettuce. Seedlings (28-d old) were subjected to drought stress by lowering the water level in the solution tub. AsA content per fresh weight of whole aerial part was measured at each water level $(0,0.5$, $1.0,2.0$, and $4.0 \mathrm{~cm}$ of airspace) after the 7-d treatment. Conditions for growth and treatments were as follows: $20{ }^{\circ} \mathrm{C}\left(68.0^{\circ} \mathrm{F}\right), 14$-h light/10-h dark photoperiod with light provided by cool-white fluorescent lamps (photosynthetic photon flux of 60/ $140 \mu \mathrm{mol} \cdot \mathrm{m}^{-2} \cdot \mathrm{s}^{-1}$ for germination/ cultivation). Vertical bar indicates SE of six replicates. Different letters beside bars indicate significant differences at $P=0.05$ (Tukey-Kramer's honestly significant difference test). See the caption of Fig. 1 for details of seedling growth and hydroponic system; $1 \mathrm{~cm}=$ $0.3937 \mathrm{inch}, 1 \mathrm{mg} / 100 \mathrm{~g}=10 \mathrm{ppm}$.

by Day 14. Fresh leaf weight remained steady for the first $7 \mathrm{~d}$, but began to decrease significantly after Day 7 (Fig. 3 , open circles). These results suggested an optimum treatment length of $7 \mathrm{~d}$. Leaf water content, an index of freshness in lettuce, ranged from $95.6 \%$ to $96.3 \%$, and did not change significantly during the stress treatments (data not shown). We observed that leaves were not wilted or hardened by the treatments, as compared with controls.

We measured other components of lettuce leaf quality under these optimized drought conditions $(7 \mathrm{~d}$ of drought treatment with $4 \mathrm{~cm}$ of airspace) (Table 1). Total polyphenol content increased significantly $(P \leq$ $0.01)$ in response to drought stress. Like AsA, polyphenol is an antioxidant. Polyphenol in drought-stressed plants was 1.50 times that of the control. Sugar content increased by 1.17 times relative to the control $(P \leq$ $0.01)$. Finally, $\mathrm{NO}_{3}{ }^{-}$nitrogen, which is an undesirable component of leafy

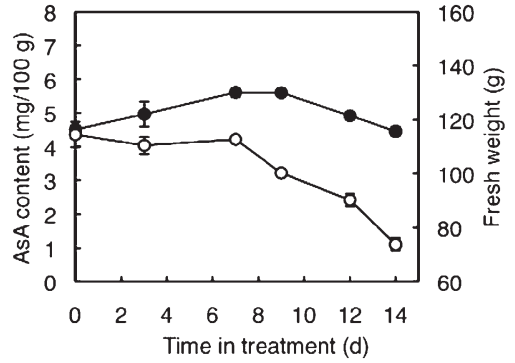

Fig. 3. Effects of duration of drought stress to lettuce roots on total Lascorbic acid (AsA) content and growth. AsA content (closed circle) and fresh weight (open circle) were measured after $0,3,7,9,12$, and $14 \mathrm{~d}$ of drought treatment. AsA content per fresh weight of aboveground part was measured at water level with $4.0 \mathrm{~cm}$ (1.57 inches) of airspace. Conditions for growth and treatments were as follows: $20{ }^{\circ} \mathrm{C}\left(68.0^{\circ} \mathrm{F}\right)$, 14-h light/ 10-h dark photoperiod with light provided by cool-white fluorescent lamps (photosynthetic photon flux of $60 / 140 \mu \mathrm{mol} \cdot \mathrm{m}^{-2} \cdot \mathrm{s}^{-1}$ for germination/cultivation). Vertical bar indicates SE of six replicates. See the caption of Fig. 1 for details of seedling growth and hydroponic system; $1 \mathrm{mg} /$ $100 \mathrm{~g}=10 \mathrm{ppm}, 1 \mathrm{~g}=0.0353 \mathrm{oz}$.

vegetables, decreased by $\approx 18 \%$ with drought treatment $(P \leq 0.01)$.

AsA content after drought treatment was measured in other leafy vegetables to determine whether dehydration stress causes similar effects as in lettuce (Fig. 4). Turnip leaf, spinach, and crown daisy all accumulated significantly higher levels of AsA in response to drought stress, with levels 1.63 , 1.44 , and 1.48 times than that of their respective controls $(P \leq 0.05)$. Although drought-stressed potherb mustard had 1.22 times as much AsA as its control, this effect was not significant $(P>0.05)$.

\section{Discussion}

In this study, we demonstrated that a novel hydroponic treatment can successfully improve the nutritional quality of some leafy vegetables with little difficulty or added expense. The roots of plants were exposed to drought stress by simply lowering the water level in the solution tub just before harvesting (Fig. 1). To our knowledge, this is the first report of an economical and practical method to improve leafy crop quality that requires no additional capital investment or operating costs for growers. 
The correlation between some antioxidants and environmental stress was reviewed by Blokhina et al. (2003). In this study, the levels of two antioxidants, AsA and polyphenol, in lettuce leaves increased significantly in response to drought stress (Fig. 2; Table 1). Some studies of soil-cultured plants have indicated that safflower and cucumber also accumulate these two compounds in response to osmotic stress induced by restricted irrigation (Yaginuma et al., 2002, 2003 ). Drought stress in the roots triggers oxidative stress in the whole plant as a result of changes in root

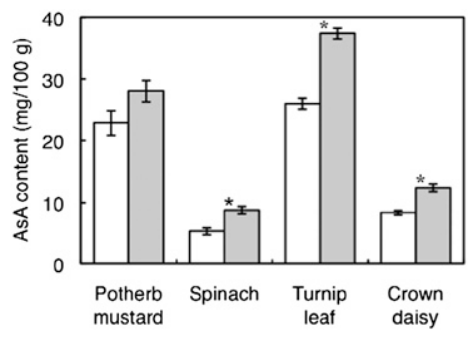

Fig. 4. Effects of drought stress to roots in leafy vegetables on total $\mathrm{L}$-ascorbic acid (AsA) content. Each seedling of 18-d-old potherb mustard, 22-d-old spinach, 21-d-old turnip leaf, and 36d-old crown daisy was subjected to drought stress by reducing the water level by $0 \mathrm{~cm}$ [control (white bar)] or $4 \mathrm{~cm}$ [1.6 inches (black bar)] for $7 \mathrm{~d}$. Conditions for growth and treatments were as follows: $20^{\circ} \mathrm{C}\left(68.0^{\circ} \mathrm{F}\right), 14-\mathrm{h}$ light/10-h dark photoperiod with light provided by cool-white fluorescent lamps (photosynthetic photon flux of $60 / 140 \mu \mathrm{mol} \cdot \mathrm{m}^{-2} \cdot \mathrm{s}^{-1}$ for germination/cultivation). Small vertical bars indicate SE of six replicates. Different letters above each bar indicate significant difference between control and treatment at $\boldsymbol{P}=\mathbf{0 . 0 5}$ (Student's $t$ test). See the caption of Fig. 1 for details of seedling growth, hydroponic system, and treatment; $1 \mathrm{mg} / 100 \mathrm{~g}=$ $10 \mathrm{ppm}$. absorption and leaf photosynthesis rates (Kitano et al., 2008). The stress might cause the aerial parts of plants to increase antioxidant substances as protection from injury by reactive oxygen species. Other antioxidant substances, such as glutathione and tocopherol, also increase in response to oxidative stress in plants (Blokhina et al., 2003; Romani et al., 2002).

Generally, there is a trade-off between growth and accumulation of antioxidants in stressed plants (Hidaka et al., 2008). However, a narrow range of stress conditions may exist that could increase antioxidant levels without retarding growth. We optimized two parameters of drought intensity: the area of roots subject to dehydration and the duration of exposure. Lettuce leaves accumulated the highest levels of AsA when there was a $4-\mathrm{cm}$ air gap between the base of the stems and the water level over a duration of $7 \mathrm{~d}$ (Figs. 2 and 3). The fresh weight of lettuce declined significantly relative to the control only after $9 \mathrm{~d}$ of drought treatment. Thus, there appears to be a threshold stress value at which benefits can be maximized without harmful effects. Kitano et al. (2008) have reported that levels of stress even slightly above the threshold value decreased growth, so determination of this threshold value is critical.

AsA content was increased by drought stress to roots early in the treatment period (Fig. 3). After Day 9, AsA levels began to decline gradually and by Day 14 had reached the same level as control plants. When plants either escape stress conditions or become acclimatized to them, they return to their baseline status (Matsui and Li, 2003). This result indicates that the antioxidant substances increased to protect the plant in the early stages of stress and decreased after exceeding the threshold for maintaining growth. Although leaf

Table 1. Effects of optimized root drought stress on total polyphenol, nitrate nitrogen, and sugar content in lettuce. Seedlings (28-d old) were subjected to reduced water levels $[$ control $=0 \mathrm{~cm}$, drought $=4 \mathrm{~cm}$ (1.6 inches) $]$ for $7 \mathrm{~d}$.

\begin{tabular}{|c|c|c|}
\hline Components $^{\mathrm{z}}$ & Control $(\text { mean } \pm \mathrm{SE})^{\mathrm{y}}$ & Drought $($ mean $\pm S E)$ \\
\hline Total polyphenol $(\mathrm{mg} / 100 \mathrm{~g})$ & $33.80 \pm 0.46$ & $51.00 \pm 1.11 * *$ \\
\hline Nitrate nitrogen $(\mathrm{mg} / \mathrm{l} 00 \mathrm{~g})$ & $387.92 \pm 10.59$ & $318.73 \pm 8.70 * *$ \\
\hline Sugar $(\%)$ & $2.42 \pm 0.07$ & $2.84 \pm 0.04 * *$ \\
\hline
\end{tabular}

Significant differences at $P=0.01$ (Student's $t$ test).

fresh weight decreased with increased duration of drought treatment, there was no significant alteration in water content among the different treatments (data not shown). Leaf water content is an indicator of palatability, such as eating texture, in leafy vegetables. Although we did not measure leaf color or leaf area, we did not observe visible differences in these parameters in droughtstressed plants. These observations suggest that the increase of valuable components did not result simply from an increase in concentration due to water loss.

Plants exposed to osmotic stress maintain leaf turgor pressure by accumulating osmolytes like sugar, minerals, and amino acids (Hasegawa et al., 2000). In our study, sugar content increased significantly in response to drought treatment in lettuce (Table 1). This might be due to osmoregulation in the plants subjected to drought stress. Hidaka et al. (2008) reported that low-temperature stress to spinach roots enhanced sugar content and decreased $\mathrm{NO}_{3}{ }^{-}$content in the shoots. Nitrate is not only harmful to human health but also causes a bitter taste in vegetables. In this study, drought treatment under conditions that increased antioxidant levels also decreased $\mathrm{NO}_{3}{ }^{-}$ content significantly (Table 1). Fennell and Markhart (1998) reported that exposing roots to low-temperature stress decreased the root hydraulic conductance. This suggests that the decrease in $\mathrm{NO}_{3}{ }^{-}$in the aerial parts of plants under drought stress can be induced by the reduction of nutrient uptake as well as by low-temperature stress. These results suggest the possibility that the drought treatment to roots could improve taste in vegetables.

We evaluated the effects of drought stress on four other leafy vegetables. The AsA content increased in turnip leaf, crown daisy, and spinach, but not in potherb mustard (Fig. 4). Plant responses to water stress are likely to be controlled by complex physiological mechanisms that differ from species to species (Xu et al., 2010). The sensitivity, response, and tolerance to stress can be different in different plant species or even in different developmental and growth stages of the same species. To achieve the highest quality foods including optimum contents of polyphenolic compounds, $\mathrm{NO}_{3}{ }^{-}$nitrogen, and sugar, stress conditions should be optimized for each 
crop species. However, the novel method presented here for hydroponic lettuce cultivation has a universal potential to increase food quality without yield reduction and at low expense.

\section{Literature cited}

Blokhina, O., E. Virolainen, and K.V. Fagerstedt. 2003. Antioxidants, oxidative damage and oxygen deprivation stress. Ann. Bot. (Lond.) 91:179-194.

Cuartero, J. and R. Fernández-Muñoz. 1999. Tomato and salinity. Sci. Hort. 78:83-125.

Fennell, A. and A.H. Markhart. 1998. Rapid acclimation of root hydraulic conductivity to low temperature. J. Expt. Bot. 49:879-884.

Fusco, D., G. Colloca, M.R. Lo Monaco, and M. Cesari. 2007. Effects of antioxidant supplementation on the aging process. Clin. Interv. Aging 2:377-387.

Gupta, S. and J. Prakash. 2008. Influence of antioxidant components on antioxidant activity of dehydrated green leafy vegetables. Food Sci. Technol. Res. 14: 104-109.

Hamilton, J.M. and J.M. Fonseca. 2010. Effect of saline irrigation water on antioxidants in three hydroponically grown leafy vegetables: Diplotaxis tenuifolia, Eruca sativa, and Lepidium sativum. HortScience 45:546-552.

Hasegawa, P.M., R.A. Bressan, J.K. Zhu, and H.J. Bohnert. 2000. Plant cellular and molecular responses to high salinity. Annu. Rev. Plant Physiol. Mol. Biol. 51:463-499.

Hidaka, K., D. Yasutake, M. Kitano, T. Takahashi, Y. Sago, K. Ishikawa, and T. Kawano. 2008. Production of high quality vegetables by applying low temperature stress to roots. Acta Hort. 801:1431-1436.

Iwai, K. 2008. Antidiabetic and antioxidant effects of polyphenols in brown alga Ecklonia stolonifera in genetically diabetic KK-A mice. Plant Foods Hum. Nutr. 63:163-169.

Johkan, M., K. Shoji, F. Goto, S. Hashida, and T. Yoshihara. 2010. Blue light-emitting diode light irradiation of seedlings improves seedling quality and growth after transplanting in red leaf lettuce. HortScience 45:1809-1814.

Kitano, M., K. Hidaka, K. Zushi, and T. Araki. 2008. Production of value-added vegetables by applying environmental stresses to roots in soil-less culture. J. Jpn. Soc. High Technol. Agr. 20:210218.

Kratky, B.A. 1993. A capillary, noncirculating hydroponic method for leaf and semi-head lettuce. HortTechnology 3: 206-207.

Kratky, B.A. 2004. A suspended pot, noncirculating hydroponic method. Acta Hort. 648:83-89.

Matsui, S. and J. Li. 2003. Environmental stress for crops and their antioxidative mechanisms. Regulat. Plant Growth Dev. 38:118-124.

Matsumoto, K., Y. Tada, H. Shimizu, and S. Shibusawa. 2009. Effect of temperature on the growth and antioxidative activity of Raphanus sativus L. Kaiware-daikon (japanese radish sprout). J. Jpn. Soc. High Technol. Agr. 21:29-34.

Nicolle, C., A. Carnat, D. Fraisse, J.L. Lamaison, E. Rock, H. Michel, P. Amouroux, and C. Remesy. 2004. Characterisation and variation of antioxidant micronutrients in lettuce (Lactuca sativa folium). J. Sci. Food Agr. 84:2061-2069.
Oh, M.M., E.E. Carey, and C.B. Rajashekar. 2009. Environmental stresses induce health-promoting phytochemicals in lettuce. Plant Physiol. Biochem. 47:578583.

Romani, A., P. Pinellia, C. Galardia, G. Sanib, A. Cimatob, and D. Heimlerc. 2002. Polyphenols in greenhouse and open-air-grown lettuce. Food Chem. 79:337-342.

Sharma, P. and R.S. Dubey. 2005. Drought induces oxidative stress and enhances the activities of antioxidant enzymes in growing rice seedlings. J. Plant Growth Regul. 46:209-221.

Sunaga, Y., H. Harada, and T. Hatanaka. 1999. Method of estimating nitrate nitrogen concentration of standing corn ( $\mathrm{Zea}$ mays L.) by nitrate nitrogen concentration in stem juice. Grassland Sci. 45:299-303.

Tabata, K., K. Oba, K. Suzuki, and M. Esaka. 2001. Generation and properties of ascorbic acid-deficient transgenic tobacco cells expressing antisense RNA for L-galactono-1,4-lactone dehydrogenase. Plant J. 27:139-148.

Takatsuji, M. 2010. Present status of completely-controlled plant factories. J. Jpn. Soc. High Technol. Agr. 22:2-7.

Xu, Z., G. Zhou, and H. Shimizu. 2010. Plant responses to drought and rewatering. Plant Signal. Behav. 5:649-654.

Yaginuma, S., T. Shiraishi, H. Ohya, and K. Igarashi. 2002. Polyphenol increases in safflower and cucumber seedlings exposed to strong visible light with limited water. Biosci. Biotechnol. Biochem. 66:65-72.

Yaginuma, S., T. Shiraishi, and K. Igarashi. 2003. Developmental transition of the flavonoid contents in safflower leaves during stress-loaded cultivation. Biosci. Biotechnol. Biochem. 67:1691-1698. 